\title{
Aún quedan posiciones por defender: Un recuento del camino recorrido por el proyecto Alfabetización Crítica
}

There are still positions to be defended:

An account of the road travelled by the Critical Literacy project

\author{
Volumen 19, Número 1 \\ Sección Especial \\ Enero-Abril \\ pp. 1-13
}

Este número se publica el 1 de enero de 2019

DOI: 10.15517/aie.v19i1.35700

Valeria Sancho Quirós

Revista indizada en REDALYC, SCIELO

Revista distribuida en las bases de datos:

LATINDEX, DOAJ, REDIB, IRESIE, CLASE, DIALNET, SHERPA/ROMEO, QUALIS-CAPES, MIAR

Revista registrada en los directorios:

ULRICH'S, $\underline{\text { REDIE}}, \underline{\text { RINACE}}, \underline{\text { OEI }}, \underline{\text { MAESTROTECA }}, \underline{\text { PREAL, }}$ 


\title{
Aún quedan posiciones por defender: Un recuento del camino recorrido por el proyecto Alfabetización Crítica
}

There are still positions to be defended:

\author{
An account of the road travelled by the Critical Literacy project
}

\section{Valeria Sancho Quirós ${ }^{1}$}

\begin{abstract}
Resumen: Alfabetización Crítica es un proyecto inscrito en la División de Educología en la Universidad Nacional de Costa Rica, compuesto desde su inicio en 2004 por un equipo de trabajo interdisciplinario. La pedagogía crítica, la sociología de la educación, la filosofía de la educación más recientemente las reflexiones en torno a la interculturalidad y las pedagogías descoloniales, entre otros saberes afines como la psicología social, han nutrido su quehacer políticamente comprometido. Este proyecto ha atravesado cuatro etapas distintas enfocadas en problemáticas coyunturales del sistema educativo, proyectándose hacia una quinta etapa en el período 2019 2021. El recorrido de Alfabetización parte de la constatación de una crisis de la educación secundaria en el país, y la consecuente disputa en torno a la lectura adecuada de sus signos. El posicionamiento en torno a los nuevos proyectos educativos que deberían erigirse, en respuesta al desgaste del modelo educativo tradicional, ha requerido un abordaje cuestionador del discurso mediático oficial que revela una agenda neoliberalizante, incompatible con el reconocimiento de los y las estudiantes en tanto sujetos políticos y epistemológicos. Rompiendo con este modelo, Alfabetización Crítica ha apostado por el ejercicio de la investigación-acción en el campo educativo. A continuación, ofrezco un breve recorrido a través de los principales momentos de este proyecto, desde sus inicios y hasta el presente, vistos a través de su producción académica.
\end{abstract}

Palabras clave: alfabetización crítica, pedagogía crítica, educación secundaria, investigación-acción participativa, neoliberalismo, interculturalidad, pedagogías descoloniales

\begin{abstract}
Critical Literacy is a Project that belongs to the Division of Educology in the National University of Costa Rica, comprised by an interdisciplinary team since its beginnings in 2004. Critical pedagogy, sociology of education, and more recently, reflections surrounding interculturalism and decolonial pedagogies, among other related branches of knowledge such as social psychology, have nurtured its work which is politically committed. This project has gone through four different stages, which focused on junctural issues of the education system, projecting itself towards a fifth stage between 2019-2021. Critical Literacy's path starts off with the acknowlegment of a crisis in secondary education, and the subsequent dispute over the adequate reading of its signs. Taking a stance over the new educational projects that should be erected, in response to the exhaustion of the traditional model, has required a critical approach of the official media discourse that reveals a neoliberalizing agenda, inconsistent with the recognition of students as political and epistemological subjects. Departing from this logic, Critical Literacy has placed its stakes in participatory-action research in the educational field. In this paper, I offer a brief walk through the Project's most important moments, since it's beginnings to present day, as seen through its academic production.
\end{abstract}

Key words: critical literacy, critical pedagogy, secondary education, participatory action research, neoliberalism, interculturalism, decolonial pedagogies

\footnotetext{
1 Investigadora del Proyecto Alfabetización Crítica, inscrito en el Centro de Docencia e Investigación en Educación, Universidad Nacional de Costa Rica.
}

Dirección electrónica: valeria.sancho90@gmail.com

Documento recibido: 6 de julio, 2018

Enviado a corrección: 2 de octubre, 2018

Aprobado: 26 de noviembre, 2018 


\section{Primera fase. Alfabetización Crítica en la Cultura Escolar, 2004-201}

Los planteamientos de Freire y Macedo (1989), en torno a una alfabetización crítica inserta en el marco de la teoría de la producción cultural, dieron su punto de partida y principal referente teórico al proyecto. Siguiendo esta línea, la alfabetización crítica se ofrece como un medio para constituir y afirmar "los momentos históricos y existenciales de la experiencia vivida que genera una cultura sometida", entendiendo este como un fenómeno eminentemente político (p.185).

A nivel metodológico, la primera fase de Alfabetización Crítica encontró un hito importante en el encuentro con la socióloga argentina Silvia Duschatzky, en 2005, en una jornada de trabajo sobre la situación de la educación secundaria en el país. Desde entonces existía prácticamente un consenso sobre la crisis que atravesaba la educación secundaria, constatable en indicadores como la llamada "deserción escolar masiva", las brechas de calidad entre el ámbito público y el privado, la desigualdad entre regiones del país, entre otros. La figura del profesional en educación en tanto funcionario público, por su parte, experimentaba una importante devaluación en el marco del paso del estado benefactor hacia el reformismo neoliberal en el país (D’Antoni y Soto, 2010).

Desde entonces los y las investigadoras del proyecto señalaron el efecto doblemente enajenante del mercado de los paquetes educacionales producidos por las industrias editoriales en series de textos prediseñados, ajenos a los contextos del estudiantado, que impedían a los y las docentes participar en tanto educadores en la planificación y desarrollo de su trabajo. Asimismo identificaron una intención de fragmentar y separar, presente a lo largo de todos los elementos del currículo nacional, que saboteaba los intentos de reunión y agrupación entre colegas (D’Antoni, Induni y Pacheco, 2005).

Según la perspectiva compartida por Duschatzky, en su visita de 2005, la complejidad del momento histórico, para el ámbito educativo, ameritaba un estudio desprovisto de esquemas pre-formados, es decir: una inserción directa en las aulas. El equipo entonces asumió una inmersión profunda en los ambientes escolares, con el objetivo de conocer las dinámicas y resistencias que podrían dar respuesta a la pregunta por los motivos de la "deserción escolar masiva".

Alfabetización Crítica hizo observaciones en aulas y entrevistas a docentes y estudiantes en diversas instituciones de educación pública, desarrollando una visión desde adentro. Lo que comprobó de manera reiterada fue "el desagrado, el dolor, el rechazo de los estudiantes en las aulas y hacia la institución educativa” (D’Antoni, Soto, Gómez y Gómez, Volumen 19 Número 1 (Enero-Abril), ISSN 1409-4703 
2007, p.74), su claro repudio al autoritarismo en las relaciones docente-estudiante, así como la insistente demanda de reconocimiento de su condición de sujetos. En cuanto a los y las docentes, el equipo les descubrió ejerciendo la función de censoras, que negaban la posibilidad de la pregunta a un estudiantado forzado a elegir entre el mutismo y la repetición de respuestas prescriptas. Las observaciones mostraron a docentes que hacían caso omiso, en el transcurso de las clases, de los conocimientos previos del estudiantado. Visto en términos cuantitativos, solo un $3 \%$ de las participaciones en clase se podían atribuir a los y las estudiantes.

La reiteración de este ejercicio vertical de la docencia, en sus efectos sistemáticos, parecía acoplarse a un "verdadero plan de desmontaje de las capacidades cognitivas" de los y las estudiantes (D’Antoni, Soto, Gómez y Gómez, 2007, p.4). La negación institucionalizada de cualquier posibilidad de producción de conocimiento por parte del estudiantado, asimismo, se reveló como una condición previa a la "deserción”. Frente a una visión patologizante, basada en la distinción de desempeños diferenciales y las mal llamadas "dificultades de aprendizaje", Alfabetización Crítica posicionó las irrupciones de los y las estudiantes como reacciones sanas de oposición al aplanamiento y el deterioro de sí (D’Antoni, Gómez, Gómez y Soto, 2011).

Los hallazgos del trabajo en aulas reafirmaron la necesidad de problematizar la posición de docente y la deslealtad de clase que se expresaba en su ejercicio del poder, frente a un estudiantado del mismo origen socioeconómico. Según palabras del equipo investigador "el educador, en la metáfora de un país con más maestros que soldados, representa exactamente eso, un soldado raso, que acata órdenes sin reflexión y es vigilado con sospechas por el resto de la jerarquía: desde los propios estudiantes y colegas, padres de familia, medios de comunicación" (D’Antoni y Pacheco, 2005, p.100); sin dejar de celebrar la presencia de docentes activamente descontentos y dispuestos a emprender un camino riesgoso hacia la transformación de los procesos de aprendizaje (D’Antoni, Gómez, Gómez, y Soto, 2011).

Las reflexiones en torno al autoritarismo en la educación pública, la desobediencia y resistencia, su relación con una formación para la ciudadanía (D’Antoni, Gómez, Gómez y Soto 2011), así como la situación de poblaciones migrantes y aborígenes en el sistema educativo (Gómez, Gómez, Pineau y Mora, 2010) también fueron llevadas a talleres, conversatorios, y jornadas de trabajo. Se ideó una estrategia para revitalizar la cultura política en la educación secundaria, que consistía en identificar las formas de resistencia 
presentes en las culturas escolares, proyectando a la construcción de un modelo pedagógico que movilizara estas formas de resistencia hacia una participación política consciente y reflexiva.

Más que resistencia en un sentido totalizador, no obstante, lo que se encontró fueron resistencias, concretas y emergente: debates éticos y políticos, vestimenta desafiante hacia el reglamento interno, reuniones de grupos o bandas con intereses comunes, pintar paredes, planificar con otros acciones de demanda de derechos, organizar y asistir a huelgas, $u$ otras actitudes más agresivas, además de la desobediencia directa (D’Antoni, Gómez, Gómez, y Soto, 2011).

Para el año 2010 el proyecto daba cuenta de "más de 90 historias de estudiantes abiertamente declarados en contra del excesivo control sobre sus cuerpos" (D'Antoni, Gómez, Gómez y Soto, 2011, p.43), destacando la existencia de un abuso constante de la autoridad. Entre los testimonios recogidos se encontraban quejas como las de Jonás de octavo año, quien señalaba que "el colegio no sirve para nada, que no ha aprendido nada para la vida, que todo lo que enseñan no funciona, nada le dicen de cómo vivir afuera, en esa selva que se come a todos" (D’Antoni, Gómez, Gómez y Soto, 2011, p.49).

En este contexto se formularon respuestas desde lo pedagógico, reivindicando en talleres con docentes la importancia de "reconocer, apreciar y cultivar" las emociones en el aula. En esta apuesta, se recuperó la concepción vigotskiana de la imaginación, entendida como la relación entre emoción e inteligencia, ubicada como una acción que trasciende los límites de la psiquis individual (D’Antoni y Malavassi, 2004)2.

En términos institucionales, y como parte del trabajo en red con ONG's, sindicatos de educadores y universidades, Alfabetización tomó posición frente a las múltiples demandas con que contaba el Ministerio de Educación Pública: casos de acoso y abuso sexual, corrupción, atropellos cotidianos... A modo de provocación, el equipo diseñó y gestionó la propuesta de una Defensoría de Estudiantes de Educación Secundaria, llevada a la Unión Europea y la Oficina de Cooperación Internacional. Esta habría de ubicarse en las Universidades en que se imparten carreras de Enseñanza, alimentándose de su recurso técnico, económico, e institucional. Sus objetivos: recoger denuncias de estudiantes y

El aporte de Vygotksy al estudio de la realidad educativa latinoamericana también fue reconocido en el congreso dedicado a Freire y Vygotski en el año 2007 cuyo décimo aniversario conmemoramos precisamente en este Congreso, gracias a una alianza estratégica con el Instituto Nacional de Investigación en Educación de la UCR. En adelante se seguirán desarrollando cursos sobre este autor, en ocasiones contando con la colaboración de la Dra. Wanda Rodríguez, y posteriormente un proyecto de memoria sobre la aplicación de su teoría a la investigación educativa latinoamericana. 
docentes hacia la institución educativa; mediar a nivel inter e intra institucional; y realizar actividades en pro de la democratización institucional y la difusión del conocimiento sobre derechos humanos y la exigibilidad de los mismos.

En cuanto al trabajo en zonas rurales, miembros del equipo asumieron acciones en Guanacaste, la provincia con mayor cantidad de personas con secundaria incompleta a nivel nacional. Las demandas de la comunidad derivaron en el acompañamiento a la oposición colectiva a un proyecto de explotación de los recursos ambientales de la cementera Holcim en las comunidades de Corral de Piedra y Roblar, dando origen a la línea de trabajo después llegó a consolidarse en la Red Epistemologías del Sur.

Además, se inscribió en el proyecto una línea que se mantiene hasta el presente: el trabajo con la comunidad indígena bribri de Kach' bli en Talamanca, que ha colaborado con Alfabetización Crítica para que el proyecto comprenda más de su cultura, saberes y prácticas, todas ellas en peligro de desaparición debido a la práctica homologadora del sistema educativo. El trabajo con la comunidad ha sido pensado en series dobles de trabajo, abarcando la producción de materiales en torno a su cultura y cosmovisión orientados a la reproducción de sus tradiciones, así como el trabajo con estudiantes de educación en la UNA que visitan la comunidad para aprender con ellas y ellos y de sus realidades, marcadas por los efectos de la desigualdad, discriminación y racismo.

\section{Segunda fase. Alfabetización Crítica en la cultura escolar: derechos humanos y sociedad 2012-2013}

En su libro "La escuela en Cuestionamiento" el equipo recogió los resultados de su trabajo en aulas. Allí Alfabetización elaboró su denuncia a la omisión reiterada, por parte de los dos gobiernos que cubrió el anterior período, de las causas estructurales de la crisis en la educación secundaria, posicionando el concepto de expulsión del sistema educativo como una expresión más adecuada que la de deserción escolar masiva, difundida entre otros por los informes del Estado de la Nación y de la Educación Costarricense. Si bien se advirtió en este libro sobre el carácter idiotizante del modelo en educación pública, se sostuvo no obstante una apuesta respecto a su importancia como "un espacio socializador y no descartable para la construcción de nuevas formas de ser y de resistir" (D’Antoni, Gómez, Gómez y Soto, 2012, p.25).

Como sur de esta fase, Alfabetización asumió la construcción de diálogos y vínculos con la docencia y la extensión, desde la Investigación-Acción Participativa, buscando 
soluciones conjuntas en pro del respeto a los derechos humanos entendidos de forma amplia y abierta. Nuevamente, se partió con Freire de que "todas las herramientas necesarias para la edificación de una nueva escuela" están "basadas en la comprensión de su origen como escuela para la dominación y el control" (D’Antoni et al., 2012, p.36). Desde esta perspectiva se llevó a cabo un proceso de diagnóstico participativo con docentes en los Liceos de Heredia, Corralillo y Nocturno León XIII, además del diseño de cursos, talleres y conferencias.

Un frente de lucha durante esta fase fue la educación universitaria, progresivamente inmersa en un "culto a la mercantilización" (D’Antoni, 2013, p.25). Alfabetización ha seguido problematizando la rankización, la formación por competencias, y la preocupación por la evaluación de la calidad, en tanto dispositivos colonizadores del conocimiento. El equipo ha advertido sobre las tendencias que orientan la producción del material humano en la universidad, hacia la formación de un sujeto técnicamente capacitado e intercambiable (D'Antoni, 2013). Frente a este proceso, se ha sostenido, urge la creación de "criterios internos de relevancia, cooperación y puesta en práctica para leer lo que suponen los imperativos de la acreditación" (D’Antoni y Gómez, 2013, p.18), comúnmente construidos sobre el parámetro de las universidades de élite, desde una jerarquía productora de exclusión y propiciadora del epistemicidio.

Siguiendo esta línea, Alfabetización atendió con preocupación al discurso gubernamental en torno a los objetivos de la educación, ahora concebida como un medio para capacitar laboralmente a grupos vulnerables, con base en las exigencias del mercado, rechazando ese discurso de doble vínculo que culpabiliza a las personas jóvenes excluidas de su propia exclusión, a la vez que les demanda una inserción cada vez más temprana a las dinámicas de reproducción capitalista. Esta tendencia se vio reflejada en la metáfora de la "deserción", que equipara al estudiantado expulsado del sistema educativo a soldados que le dan la espalda a la patria (D'Antoni, 2008), y el uso del nombre despreciativo de "los ninis", empleado en informes del Estado de la Educación para hacer referencia a quienes tendrían derecho a estar en el colegio pero no están allí, y tampoco están insertos en el mercado laboral (Herra, s.f.).

Vinculado a lo anterior el equipo abordó el tema del sonado "bullying", ubicándolo como un "discurso codificante del poder, simplificador de fenómenos asociados a la violencia estructural y escolar" (D’Antoni y Gómez, 2015, p.32). El autoritarismo y sus formas de ser 
legitimado se propusieron en este contexto como parte de un problema fundamental en el abordaje de los derechos humanos en el sistema educativo.

En esta fase inicia además una reflexión en torno a la interculturalidad, entendida como una apuesta política por la diversidad. Alfabetización ubicó el acceso diferencial a la educación y la normalización del otro a través del currículo como factores que atentan contra la diversidad en el sistema educativo. La falta de cobertura, así como de una oferta educativa de calidad y pertinente, adaptada a la realidad de los y las niñas en Talamanca Bribri, destacó como una de las omisiones más ruidosas por parte del MEP y del Tercer Estado de la Educación. Se concluyó que si bien la escuela tradicional "se declara discursivamente intercultural", esta "no soporta al verdadero otro, cuando surgen las distinciones las considera un problema a superar", pues "quien no quiera ser civilizado no será sujeto de educación" (Gómez, 2013, p.5).

\section{Tercera fase. Pedagogías críticas de lo educativo, los medios y las redes sociales, 2014-2015}

Durante esta fase el equipo hizo énfasis en las temáticas educativas abordadas por medios de comunicación, y las representaciones, discursos e imágenes sobre la educación que difundían. El estudio de tres documentos, la Memoria Institucional del MEP 2006-2014, el Manual para la Gestión de la Coordinación con la Empresa, y la Revisión de Destrezas más allá de la Escuela en Costa Rica, reveló que para el caso costarricense no atendemos a una privatización de la educación en el sentido "tradicional" de la palabra. Al contrario, el sector empresarial se beneficia de la mano de obra producida por la modalidad técnica de educación, a la cual se destinó el principal incremento presupuestal. Los nuevos colegios técnicos profesionales, que empezaron a operar bajo el Modelo de Educación Basada en Normas de Competencias, reflejan este aprovechamiento del sector público para la acumulación de capital privado. Ante este escenario se evidenció la necesidad urgente de articular alianzas entre el estudiantado, las organizaciones docentes, los movimientos sociales y el sector académico crítico para la oposición a las reformas utilitaristas y mercantilizantes de la educación (Rojas, 2015).

Durante esta fase miembros Alfabetización Crítica iniciaron un trabajo desde la perspectiva de las redes solidarias de investigación nacional e internacional que derivó en la articulación del Proyecto Epistemologías del Sur: una red integrada por investigadores e investigadoras provenientes de la Escuela de Sociología, el Instituto de Estudios en 
Población, la Escuela de Filosofía y la División de Educología, de la Universidad Nacional de Costa Rica. La Red partió del trabajo, asumido desde la Investigación-Acción-Participativa, en torno a las problemáticas enfrentadas por comunidades rurales e indígenas, que se había iniciado al interior de Alfabetización Crítica. Desde entonces, se ha sostenido en su compromiso con la investigación interdisciplinar, en interlocución con la comunidad, alrededor de denominadores comunes como el interés por los derechos humanos, el territorio y la educación.

Finalmente, se continuó durante esta fase del proyecto el trabajo de giras educativas y recolección de relatos bribri en Kach' bli, Talamanca.

\section{Cuarta etapa. Desigualdades, educabilidad y metodologías participativas de investigación, 2016-2018.}

Para esta etapa la principal preocupación del equipo ha girado en torno a la naturalización del modelo de acumulación y construcción de desigualdades que caracteriza a la región centroamericana, y su correlato socioeducativo. Siguiendo a Baquero (2002) los umbrales de "educabilidad" fueron problematizados como presunciones en torno a las formas de inclusión, exclusión y relaciones de poder en los dispositivos institucionales asociados a lo educativo (Baquero, 2002).

En este sentido el equipo atendió a la mediatización del discurso sobre la educación dual, una modalidad educativa que supone un desplazamiento progresivo de la institución educativa a la práctica profesional en la empresa, promovida como respuesta al fracaso del modelo educativo tradicional. Con este motivo se organizó en 2016 un Foro, mientras que en reflexiones sucesivas se enumeraron diversas objeciones a este modelo, tales como la distancia entre el ambiente de trabajo y las culturas jóvenes, la falta de compromiso de las empresas para con la diversidad funcional y los grupos expulsados del sistema educativo, y la dificultad de empatar la educación para el desarrollo sostenible con una formación acorde a las lógicas empresariales (D’Antoni, 2016).

Los ejes de desigualdades y educabilidad a su vez se pusieron en diálogo con la elaboración de metodologías participativas en el trabajo intercultural, desarrollándose talleres en torno a la Investigación-Acción Participativa, la Pedagogía Crítica y la Educación intercultural Indígena; publicándose una serie de libros: uno en que se ofrece una guía sobre el desarrollo de la Investigación-Acción Participativa (Mora y Gómez, 2017); uno sobre el relato más allá del libro como dispositivo cultural (Gómez y Mora, 2018); otro en el aula 
universitaria en que, mediante la metodología participativa varios estudiantes de la UNA escriben una obra sobre cómo indisciplinar la didáctica (Gómez, Mata y Ureña, 2018). Además se ha recopilado historias bribris contadas por dos Awápa (médicos tradicionales y especialistas en sabiduría y cultura bribri), que se proyecta reproducir en formato libro (en proceso de elaboración).

\section{Quinta etapa. Hacia un abordaje descolonizador del género y la diversidad sexual 2019-2021}

En esta etapa la pedagogía descolonial se dibuja como horizonte, una opción que se asume en un contexto nacional que nos obliga a atender a la colonialidad del género en tanto productora de exclusiones y violencias en la educación pública y toda la sociedad. Recurrimos a una reactualización de Freire a través de las pedagogías descoloniales, ahora ocupándonos no solo de la lucha de clases, sino de un esfuerzo descolonizador desde los pueblos y comunidades racializadas (Walsh, 2013). La pedagogía descolonial feminista se nutre de la freireana pedagogía crítica, y la Investigación-Acción Participativa de Fals Borda, extendiendo no obstante su praxis hacia el cuestionamiento y ruptura con respecto a la colonialidad del género como originaria de la exclusión social y subordinación de las mujeres en toda la región (Espinosa, Gómez, Lugones y Ochoa, 2013).

Aquí seguimos a Segato (2016) quien ha argumentado que, lejos de constituir un tema periférico, el rechazo a lo que se ha llamado "ideología de género" -cuya capacidad de convocatoria fue visibilizada por el cierre de 14 escuelas de San Carlos en protesta a la implementación de los Programas de Estudio de Educación para la Afectividad y la Sexualidad del MEP en 2017- da cuenta de la centralidad de la cuestión de género para el sostenimiento del orden neoliberal, tal y como se va imponiendo en la región más desigual del mundo. La educación sexual, en este contexto, se ha visibilizado como un tema de envergadura inclusive en lo concerniente a las recientes elecciones presidenciales.

En un contexto en que la educación diversificada muestra las menores tasas de escolaridad del país (45,8\% en 2016), la falta de acceso a educación sexual integral se visibiliza como un mecanismo de expulsión, que tiene como su efecto necesario la feminización de la pobreza y la consecuente precarización de la vida de las mujeres. No obstante el rechazo a los Programas muestra como su principal trasfondo el temor de algunos sectores a la flexibilización del binomio hombre-mujer, que supone esferas de vida 
separadas, y una división sexual del trabajo (Brown, 2010) atravesada por la "configuración diferencial de prestigio y poder" (Segato, 2016).

Sostenemos que los temores infundados de los sectores conservadores surgen de una intuición acertada, a saber, que el reconocimiento de los factores sociales y subjetivos relacionados a la sexualidad y afectividad pueden derivar en la desestabilización de las estructuras económicas y culturales que perpetúan la desigualdad de género, al suponer destinos evolutivos socialmente separados y super-jerarquizados.

Además señalamos que la disputa en torno a la posibilidad de reflexionar sobre los roles de género no es ajena respecto a las 20.421 situaciones violentas reportadas al MEP durante el último año (Cerdas, 2018), ni al sonado problema del bullying anteriormente abordado en el marco del proyecto. En ese momento el autoritarismo y sus formas de ser legitimado se propusieron como parte de un problema fundamental en el abordaje de los derechos humanos en el sistema educativo (D'Antoni y Gómez, 2013), sin embargo, las manifestaciones machistas, racistas y homófobas del bullying y su relación con estructuras de poder patriarcales y coloniales no han sido aún problematizadas

Se defiende en esta etapa del proyecto la necesidad de un análisis que reconozca un lugar central a la cuestión de género como factor estructurante de la actual crisis educativa, y que desarrolle metodologías para la alfabetización crítica en lo que respecta a la desigualdad de género y la discriminación por orientación sexual e identidad sexual. Se proyecta llevar estas metodologías directamente a las aulas, posicionando pedagogías que retoman lo lúdico, lo afectivo, lo vincular y lo genealógico como formas necesarias -aunque históricamente devaluadas- de producción de conocimiento. A la vez, se apuesta por la educación intercultural como propuesta para la transformación de nuestras concepciones sobre el género, el saber, y la historia.

\section{A modo de cierre:}

El quehacer de Alfabetización Crítica se alimenta de la futuridad esperanzadora instilada por la propuesta freireana en la educación (Freire, 1996). Frente a la razón pragmática y el fatalismo de la escuela idiotizante neoliberal (D’Antoni, Gómez, Gómez y Soto, 2012), atendemos al resurgimiento de la insumisión histórica a través de las pedagogías descoloniales y feministas. El recurso a la memoria de larga duración, que traza el conocimiento en sus orígenes geohistóricos y geopolíticos, mostrando su centralidad en la organización del sistema-mundo capitalista, es fundamental para la desideologización de un 
presente desigual y cosificador de la vida. El desaprendizaje de la razón colonial/moderna, fundada en los binarismos dicotómicos y jerarquizantes en torno a la civilización-barbarie, razón-emoción, mente-cuerpo, y las diferenciales de género y raza se perfila, de cara a la quinta etapa del proyecto, como condición necesaria para la apertura de un futuro en que la educación se realice en su vocación humanizante.

\section{Referencias}

Baquero, Ricardo. (2002). La educabilidad como problema político. En seminario Permanente de investigación de la Maestría en Educación de la UdeSA. Argentina. Recuperado de http://hdl.handle.net/10908/772

Brown, Wendy. (2010). Regulating aversion: Tolerance in the age of the empire. Estados Unidos Princeton University Press

Cerdas, Daniela. (28 de enero de 2018). Alumnos de 6 de cada 10 colegios lidian con golpes, amenazas y robos. La Nación. Recuperado de https://www.nacion.com/elpais/educacion/alumnos-de-6-de-cada-10-colegios-lidian-congolpes/OXOCEOPZLJCETJIGEWZNTAE6YA/story/

D’Antoni, Maurizia. y Malavassi, Humberto. (2004). Inteligencias y Creatividad: Teoría, Metáfora y Vivencia. Ensayos Pedagógicos, 3(1), 91-103.

D’Antoni, Maurizia., Induni, Gina., y Pacheco, Xenia. (2005). Crisis en la educación secundaria: ¿Reproducir o transformar? Educare, 8, 91-10.

D’Antoni, Maurizia, Soto, José, Gómez, Juan y Gómez, Luis. (2007). Escenarios Investigativos desde la pedagogía crítica: culturas colegiales, juveniles y políticas. En I Encuentro Internacional de Investigación Educativa El aporte de Lev Vygotsky y Paulo Freire. Facultad de Educación, Universidad de Costa Rica. San José, Costa Rica.

D’Antoni, Maurizia. (2008). Nuevas propuestas pedagógicas y el papel social del docente. Ensayos Pedagógicos (4), 11-26.

D’Antoni, Maurizia. y Soto, José. (2010). Experiencias de investigación en la secundaria: una visión desde adentro. Temas de Nuestra América, 26(49). Recuperado de http://www.revistas.una.ac.cr/index.php/tdna/article/view/3717

D’Antoni, Maurizia, Gómez, Juan., Gómez, Luis. y Soto, José. (2011). Desobediencia y resistencia de jóvenes que se hacen sujetos: el caso de enseñanza colegial costarricense. Revista Ensayos Pedagógicos, 6(1), 37-54.

D’Antoni, Maurizia., Gómez, Juan., Gómez, Luis. y Soto, José. (2012). La escuela en cuestionamiento. Diálogos sobre la resistencia escolar en procesos pedagógicos emergentes. San José, Costa Rica: Arlekín. 
D’Antoni, Maurizia. (2013). Educación superior en Costa Rica: ¿"Rankización” y descolonización? Educare, 17(3), 21-40.

D’Antoni, Maurizia. y Gómez, Luis. (2013). Repensar la universidad: reflexiones fronterizas para descolonizar prácticas entre el giro decolonial y la pedagogía crítica [Ensayo inédito].

D’Antoni, Maurizia., Gómez, Luis. (2015). "Violencia, bullying y autoritarismo en la escuela secundaria". En Reflexiones desde la investigación socio-educativa en contextos de exclusión. San José, Costa Rica: INIE.

D’Antoni, Maurizia. (2016). Educación Dual como una paradoja: 10 razones para indicar la urgencia del debate sobre la educación dual en Costa Rica [Artículo inédito].

Espinosa, Yuderkis., Gómez, Diana., Lugones, María. y Ochoa, Karina. (2013). Reflexiones pedagógicas en torno al feminismo descolonial: Una conversa en cuatro voces. En Walsh (ed). Pedagogías decoloniales: Prácticas insurgentes de resistir, (re)existir y (re)vivir. Ecuador: ediciones Abya Yala.

Freire, P. y Macedo D. (1989). Alfabetización. Lectura de la palabra y lectura de la realidad En S. Horvath (Trad.). Barcelona, España: Paidós; Ministerio de Educación y Ciencia.

Freire, Paulo. (1996) Pedagogía de la esperanza. Madrid, España: Siglo XXI Editores.

Gómez, Juan. y Mora, Mainor. (2018). El libro como dispositivo cultural: Algunos criterios de ingreso al problema del orden informacional contemporáneo. San José, Costa Rica: Arlekín.

Gómez, Juan., Mata, Adrián. y Ureña, Omar (Eds.). (2018). Indisciplinando la didáctica: la enseñanza de la filosofía en Costa Rica. En Ensayos Pedagógicos. Recuperado de http://www.revistas.una.ac.cr/index.php/ensayospedagogicos/article/view/10862/1370 $\underline{4}$

Gómez, Luis. y Gómez, Juan. (2011). Elementos teóricos y prácticos de la pedagogía crítica. Más allá de la educación, metáfora escena y experiencia. Praxis, 66, 181-190.

Gómez, Luis., Gómez, Juan., Pineau, Fanny. y Mora, András. (2010). La educación desde los pueblos indígenas de Bolivia. Análisis del proyecto de Nueva Ley de Educación "Avelino Siñani y Elizardo Pérez" como resistencia frente a la educación para la globalización neoliberal. Temas de Nuestr américa, 26(48), 117-136

Herra, Ernesto. (s.f.). Exclusión social como práctica pedagógica en la economía mundo capitalista. El caso del abandono del sistema educativo formal costarricense por jóvenes entre los 12 y los 18 años de edad. Recuperado de https://www.academia.edu/3371036/Exclusi\%C3\%B3n social como pr\%C3\%A1ctica pedag\%C3\%B3gica en la econom\%C3\%ADa mundo capitalista 
Mora, Mainor. y Gómez, Juan. (2017). Algunos fundamentos de investigación social. Heredia, Costa Rica: EUNA.

Rojas, Vinicio. (2015). Hacia una Educación Secundaria al Servicio del Mercado: Reflexiones en torno al papel de la Educación Técnica Profesional en Costa Rica. En XXX Congreso Alas, San Jose, Costa Rica.

Segato, Rita. (2016). La guerra contra las mujeres. Madrid, España: Traficantes de sueños.

Walsh, Catherine. (2013). Lo pedagógico y lo decolonial: entretejiendo caminos. En Walsh (ed). Pedagogías decoloniales: Prácticas insurgentes de resistir, (re)existir y (re)vivir . Ecuador: ediciones Abya Yala. 